\title{
MULTIPLE POLYLOGARITHMS, CYCLOTOMY AND MODULAR COMPLEXES
}

\section{A. B. Goncharov}

\section{Introduction}

1. Multiple polylogarithms. We define them by the power series expansion:

$$
L i_{n_{1}, \ldots, n_{m}}\left(x_{1}, \ldots, x_{m}\right)=\sum_{0<k_{1}<k_{2}<\ldots<k_{m}} \frac{x_{1}^{k_{1}} x_{2}^{k_{2}} \ldots x_{m}^{k_{m}}}{k_{1}^{n_{1}} k_{2}^{n_{2}} \ldots k_{m}^{n_{m}}} .
$$

Here $w:=n_{1}+\ldots+n_{m}$ is called the weight and $m$ the depth.

These power series are convergent for $\left|x_{i}\right|<1$, and can be continued analytically via the iterated integral presentation given by theorem 2.1.

The power series (1) generalize both the classical polylogarithms $L i_{n}(x)$ $(m=1)$, and multiple $\zeta$-values $\left(x_{1}=\ldots=x_{m}=1\right)$ :

$$
\zeta\left(n_{1}, \ldots, n_{m}\right):=\sum_{0<k_{1}<k_{2}<\ldots<k_{m}} \frac{1}{k_{1}^{n_{1}} k_{2}^{n_{2}} \ldots k_{m}^{n_{m}}} \quad n_{m}>1 .
$$

The multiple $\zeta$-values were invented and studied by Euler [E] and then forgotten. They showed up again in such different subjects as quantum groups [Dr] (the Drinfeld associator), Zagier's studies [Z1-2], the Kontsevich integrals for Vassiliev knot invariants, mixed Tate motives over Spec $\mathbb{Z}$ [G1-2], and, recently, in computations in quantum field theory $[\mathrm{B}],[\mathrm{Kr}]$.

The multiple polylogarithms were studied in [G1-4]. In this paper we investigate them at $N$-th roots of unity: $x_{1}^{N}=\ldots=x_{m}^{N}=1$. Notice that $L i_{1}(x)=-\log (1-x)$, so if $\zeta_{N}$ is a primitive $N$-th root of 1 , then $L i_{1}\left(\zeta_{N}\right)$ is a logarithm of a cyclotomic unit in $\mathbb{Z}\left[\zeta_{N}, N^{-1}\right]$. In general the supply of numbers we get coincides with the linear combinations of multiple Dirichlet $L$-values

$$
L\left(\chi_{1}, \ldots, \chi_{m} ; n_{1}, \ldots, n_{m}\right):=\sum_{0<k_{1}<k_{2}<\ldots<k_{m}} \frac{\chi_{1}\left(k_{1}\right) \ldots \chi_{m}\left(k_{m}\right)}{k_{1}^{n_{1}} k_{2}^{n_{2}} \ldots k_{m}^{n_{m}}} .
$$

They are periods of mixed Tate motives over the scheme $S_{N}:=\operatorname{Spec} \mathbb{Z}\left[\zeta_{N}\right]\left[\frac{1}{N}\right]$ (see s. 11 of [G2] and [G4]).

To study these numbers we introduce some tools from homological algebra (cyclotomic and dihedral Lie algebras, modular complex for $\left.G L_{m}(\mathbb{Z})\right)$ and geometry

Received June 6, 1998.

The author was supported by NSF grant DMS-9500010. 
(a realization of the modular complex in the symmetric space $S L_{m}(\mathbb{R}) / S O_{m}$ ). To motivate them we start from a conjecture.

2. Multiple polylogarithms at roots of unity and the cyclotomic Lie algebras. Let $\mathcal{Z}_{\leq w}(N)$ be the $\mathbb{Q}$-vector space spaned by the numbers

$$
\bar{L} i_{n_{1}, \ldots, n_{m}}\left(\zeta_{N}^{\alpha_{1}}, \ldots, \zeta_{N}^{\alpha_{m}}\right):=(2 \pi i)^{-w} L i_{n_{1}, \ldots, n_{m}}\left(\zeta_{N}^{\alpha_{1}}, \ldots, \zeta_{N}^{\alpha_{m}}\right) .
$$

Here we may take any branch of $L i_{n_{1}, \ldots, n_{m}}\left(x_{1}, \ldots, x_{m}\right)$. Then $\mathcal{Z}(N):=$ $\cup \mathcal{Z}_{\leq w}(N)$ is an algebra bifiltered by the weight and by the depth. For example:

$$
L i_{m}(x) \cdot L i_{n}(y)=\sum_{k_{1}, k_{2}>0} \frac{x^{k_{1}} y^{k_{2}}}{k_{1}^{m} k_{2}^{n}}=L i_{m, n}(x, y)+L i_{m+n}(x y)+L i_{n, m}(y, x) .
$$

(To prove this split the sum over $k_{1}<k_{2}, k_{1}=k_{2}$ and $k_{1}>k_{2}$ ).

Denote by $U C \bullet$ the universal enveloping algebra of a graded Lie algebra $C$ • Let $U C_{\bullet}^{\vee}:=\oplus_{n \geq 0}(U C)_{n}^{\vee}$ be its graded dual. It is a commutative Hopf algebra.

Conjecture 1.1. There exists a graded Lie algebra $C \bullet(N)$ over $\mathbb{Q}$ such that one has an isomorphism

$$
\mathcal{Z}(N)=U C_{\bullet}(N)^{\vee}
$$

of filtered by the weight on the left and by the degree on the right algebras.

b) $H_{(n)}^{1}\left(C_{\bullet}(N)\right)=K_{2 n-1}\left(\mathbb{Z}\left[\zeta_{N}\right]\left[\frac{1}{N}\right]\right) \otimes \mathbb{Q}$.

c) $C_{\bullet}(1)$ is free graded Lie algebra.

Here $H_{(n)}$ is the degree $n$ part of $H$. Notice that $H_{(n)}^{1}\left(C_{\bullet}(N)\right)$ is dual to the space of degree $n$ generators of the Lie algebra $C_{\bullet}(N)$.

A construction of the Lie algebra $C_{\bullet}(N)$ using the Hodge theory will be outlined in s. 3.2. It can be used to deduce conjecture 1.1 from some standard (but extremely dificult!) conjectures in arithmetic algebraic geometry. The simplest abelian quotient $C_{1}(N)$ of $C_{\bullet}(N)$ is the group of cyclotomic units in $\mathbb{Z}\left[\zeta_{N}, N^{-1}\right]$, tensored by $\mathbb{Q}$. We call $C \bullet(N)$ the cyclotomic Lie algebra of level $N$, and suggest that the "higher cyclotomy theory" should study its properties.

\section{Examples.}

i) Let $N=1$. Then by the Borel theorem the only nontrivial modulo torsion $K$-groups are $K_{4 n+1}(\mathbb{Z})$, which have rank 1 and correspond to $\bar{\zeta}(2 n+1)$ via the regulator map.

ii) $N=2$ : the generators should correspond to $(2 \pi i)^{-1} \log 2, \bar{\zeta}(3), \bar{\zeta}(5), \ldots$

iii) If $N>2, n>1$ one has $\operatorname{dim} K_{2 n-1}\left(\mathbb{Z}\left[\zeta_{N}, N^{-1}\right]\right) \otimes \mathbb{Q}=\frac{\varphi(N)}{2}$ and the space of generators should correspond to the span over $\mathbb{Q}$ of $\bar{L} i_{n}\left(\zeta_{N}^{\alpha}\right)$. 
Remark. Let $\pi_{1}^{(l)}\left(\mathbb{P}^{1} \backslash\{0,1, \infty\}\right)$ be the l-adic completion of the fundamental group. One has canonical homomorphism

$$
\varphi^{l}: \operatorname{Gal}(\overline{\mathbb{Q}} / \mathbb{Q}) \longrightarrow \operatorname{Out}_{1}^{(l)}\left(\mathbb{P}^{1} \backslash\{0,1, \infty\}\right) .
$$

It was studied by P. Deligne, Y. Ihara and others (see [Ih] and references there). Conjecture 1.1 for $N=1$ is closely related to some conjectures/questions of P.Deligne $[\mathrm{D}]$ about the image of the map (7) and V. Drinfeld [Dr] about the structure of the pronilpotent version of the Grothendieck-Teichmuller group.

The left hand side of (6) has an additional structure: the depth filtration. To study it we proceed as follows.

3. The dihedral Lie coalgebra and modular complexes. Let $Z_{\bullet, \bullet}(N)$ be associate graded quotient with respect to the weight and the depth filtrations of the algebra $\mathcal{Z}(N)$. We reduce it further introducing the bigraded $\mathbb{Q}$-space

$$
\bar{Z}_{\bullet, \bullet}(N):=\frac{Z_{\bullet, \bullet}(N)}{\left(Z_{>0,>0}(N)\right)^{2}} .
$$

The multiple polylogarithms are multivalued functions, however it is easy to show that the projection of $(4)$ to $\bar{Z} \bullet, \bullet(N)$ does not depend on the branch we choose. So $\bar{Z}_{w, m}(N)$ is the quotient of the $\mathbb{Q}$-space generated by the numbers (4) of weight $w$ and depth $m$ modulo the subspace generated by the lower weight and depth numbers, and also by the products of numbers (4) of total weight $w$.

Here is our strategy for investigation of $\operatorname{dim}_{\mathbb{Q}} \bar{Z}_{w, m}(N)$. Let $\mu_{N}$ be the group of $N$-th roots of unity. In the section 3 a Lie coalgebra $\mathcal{D} \bullet \bullet \cdot\left(\mu_{N}\right)$, called the dihedral Lie coalgebra, is explicitely constructed. Namely, the generators of the $\mathbb{Q}$-vector space $\mathcal{D}_{w, m}\left(\mu_{N}\right)$ correspond to the projections of the numbers (4) to $\bar{Z}_{w, m}(N)$, and the defining relations reflect the known $\mathbb{Q}$-linear relations between these numbers. We prove that $\bar{Z}_{w, m}(N)$ is a quotient of $\mathcal{D}_{w, m}\left(\mu_{N}\right)$ (theorem 7.1). A really new data is the cocommutator map $\delta: \mathcal{D}_{\bullet, \bullet}\left(\mu_{N}\right) \longrightarrow \Lambda^{2} \mathcal{D} \bullet \bullet \bullet\left(\mu_{N}\right)$. The dihedral Lie coalgebra is bigraded by the weight and the depth.

We want to understand the cohomology of the Lie coalgebra $\mathcal{D}_{\bullet, \bullet}\left(\mu_{N}\right)$. Here is the standard cochain complex computing the cohomology of $\mathcal{D} \bullet, \bullet\left(\mu_{N}\right)$ :

$$
\mathcal{D} \bullet, \bullet\left(\mu_{N}\right) \stackrel{\delta}{\longrightarrow} \Lambda^{2} \mathcal{D} \bullet, \bullet\left(\mu_{N}\right) \stackrel{\delta \wedge i d-i d \wedge \delta}{\longrightarrow} \Lambda^{3} \mathcal{D} \bullet, \bullet\left(\mu_{N}\right) \longrightarrow \ldots
$$

The first arrow is the cocommutator map, and the others obtained via the Leibniz rule. This complex is bigraded by the weight and depth. Let $\left(\Lambda^{*} \mathcal{D}\left(\mu_{N}\right)\right)_{w, m}$ be the subcomplex of the weight $w$ and depth $m$. It is easy to prove that

$$
\mathcal{D}_{w, 1}\left(\mu_{N}\right)=K_{2 w-1}\left(\mathbb{Z}\left[\zeta_{N}, N^{-1}\right]\right) \otimes \mathbb{Q} \quad \subset \quad H_{(w)}^{1}\left(\mathcal{D}_{\bullet}, \bullet(N)\right) .
$$

We construct a certain length $m-1$ complex of $G L_{m}(\mathbb{Z})$-modules $M_{(m)}^{*}$, called the rank $m$ modular complex. For $m=2$ it is identified with the chain complex of the classical modular triangulation of the hyperbolic plane: 


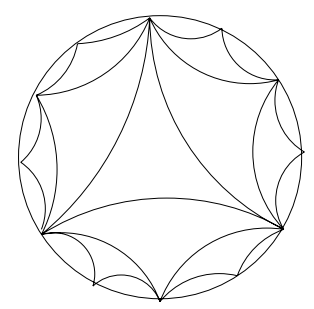

Let $V_{m}$ be the standard $m$-dimensional representation of $G L_{m}$. Denote by $\Gamma_{1}(N ; m)$ the subgroup of $G L_{m}(\mathbb{Z})$ consisting of matrices whose last row is congruent to $(0, \ldots, 0,1)$ modulo $N$.

Theorem 1.2. a) There exists a surjective morphism of complexes

$$
\mu(N)_{w, m}^{*}: \quad S^{w-m} V_{m} \otimes_{\Gamma_{1}(N ; m)} M_{(m)}^{*} \quad \longrightarrow \quad\left(\Lambda^{*} \mathcal{D}\left(\mu_{N}\right)\right)_{w, m}
$$

b) It is an isomorphism if $N=1$, or if $N$ is prime and $w=m$.

A special case of this theorem for $m=2, w=2$ was proved in [G3]. The case $N=1, m=2, w$ is arbitrary was considered in [G1] and in s. 4 of [G3].

We prove in section 6 that the modular complex of rank 3 is (essentially) quasiisomorphic to the Voronoi complex of the symmetric space $S L_{3}(\mathbb{R}) / S O_{3}$. Using these results we can estimate from above $\operatorname{dim} \bar{Z}_{w, m}(N)$ for $m \leq 3$.

4. Applications to multiple $\zeta$-values. Theorem 1.2 together with the relation between the modular and Voronoi complexes for $G L_{2}$ and $G L_{3}$ mentioned above lead to the following result.

\section{Theorem 1.3.}

$$
\begin{aligned}
& H_{(w)}^{i}\left(\mathcal{D}_{\bullet}, 2(1)\right)=H^{i-1}\left(G L_{2}(\mathbb{Z}), S^{w-2} V_{2}\right) \quad i=1,2 \\
& H_{(w)}^{i}\left(\mathcal{D}_{\bullet}, 3(1)\right)=H^{i}\left(G L_{3}(\mathbb{Z}), S^{w-3} V_{3}\right) \quad i=1,2,3
\end{aligned}
$$

This allows us to compute the Euler characterisitc of complexes $\left(\Lambda^{*} \mathcal{D}(1)\right)_{w, m}$ for $m=2,3$. Then we find $\operatorname{dim}_{\mathbb{Q}} \mathcal{D}_{w, m}(1)$ for $m=2,3$ by induction using as a starting point (9), which boils down to

$$
\operatorname{dim}_{\mathbb{Q}} \mathcal{D}_{w, 1}(1)= \begin{cases}1 & w: \text { odd } \\ 0 & w: \text { even }\end{cases}
$$

Theorem 1.4. a) If $w$ is odd, then $\operatorname{dim} \bar{Z}_{w, 2}(1)=\operatorname{dim} \mathcal{D}_{w, 2}(1)=0$.

b) If $w$ is even then $\operatorname{dim} \bar{Z}_{w, 2}(1) \leq \operatorname{dim} \mathcal{D}_{w, 2}(1)=\left[\frac{w-2}{6}\right]$.

The part a) goes back to Euler, b) was discovered by Zagier [Z2].

Theorem 1.5. a) If $w$ is even, then $\operatorname{dim} \bar{Z}_{w, 3}(1)=\operatorname{dim} \mathcal{D}_{w, 3}(1)=0$.

b) If $w$ is odd then

$$
\operatorname{dim} \bar{Z}_{w, 3}(1) \leq \operatorname{dim} \mathcal{D}_{w, 3}(1)=\left[\frac{(w-3)^{2}-1}{48}\right]
$$


Numerical calculations of multiple $\zeta$ 's by Zagier (considerebly extended by Broadhurst in $[\mathrm{B}]$ ) suggested that the estimate (13) is exact. Our results about "motivic" multiple $\zeta$ 's allow to deduce this from standard conjectures. The details will appear elsewhere.

Remark. The philosophy of mixed motives was the main driving force for us. However constructions and proofs of this paper are "elementary", i.e. do not use motives. The mixed motives/Hodge structures show up only in s. 3.2 to outline the construction of the cyclotomic Lie algebra, but we do not use s. 3.2 in the rest of the paper, so the reader may skip it.

\section{Properties of multiple polylogarithms}

\section{Iterated integral presentation. Set}

$$
\begin{aligned}
& \int_{0}^{a_{n+1}} \frac{d t}{a_{1}-t} \circ \ldots \circ \frac{d t}{a_{n}-t}:=\int_{0 \leq t_{1} \leq \ldots \leq t_{n} \leq a_{n+1}} \frac{d t_{1}}{a_{1}-t_{1}} \wedge \ldots \wedge \frac{d t_{n}}{a_{n}-t_{n}} \\
& I_{n_{1}, \ldots, n_{m}}\left(a_{1}: \ldots: a_{m}: a_{m+1}\right):= \\
& \int_{0}^{a_{m+1}} \underbrace{\frac{d t}{a_{1}-t} \circ \frac{d t}{t} \circ \ldots \circ \frac{d t}{t}}_{n_{1}} \circ \ldots \underbrace{\frac{d t}{a_{m}-t} \circ \frac{d t}{t} \circ \ldots \circ \frac{d t}{t}}_{n_{m}}
\end{aligned}
$$

The following theorem is the key to properties of multiple polylogarithms.

Theorem 2.1.

$$
L i_{n_{1}, \ldots, n_{m}}\left(x_{1}, \ldots, x_{m}\right)=I_{n_{1}, \ldots, n_{m}}\left(1: x_{1}: x_{1} x_{2}: \ldots: x_{1} \ldots x_{m}\right) .
$$

The proof is very easy: develope $d t /\left(a_{i}-t\right)$ into a geometric series and integrate. If $x_{i}=1$ we get the Kontsevich formula.

2. Relations. . The double shuffle relations. Set

$$
\begin{aligned}
& \operatorname{Li}\left(x_{1}, \ldots, x_{m} \mid t_{1}, \ldots, t_{m}\right):=\sum_{n_{i} \geq 1} L i_{n_{1}, \ldots, n_{m}}\left(x_{1}, \ldots, x_{m}\right) t_{1}^{n_{1}-1} \ldots t_{m}^{n_{m}-1} \\
& I\left(a_{1}: \ldots: a_{m}: a_{m+1} \mid t_{1}, \ldots, t_{m}\right):= \\
& \sum_{n_{i} \geq 1} I_{n_{1}, \ldots, n_{m}}\left(a_{1}: \ldots: a_{m}: a_{m+1}\right) t_{1}^{n_{1}-1} \ldots t_{m}^{n_{m}-1} \\
& I\left(a_{1}: \ldots: a_{m}: a_{m+1} \mid t_{1}, t_{1}+t_{2}, \ldots, t_{1}+\ldots+t_{m}\right)
\end{aligned}
$$

Let $\Sigma_{k, n-k}$ be the subset of permutations of $n$ letters $\{1, \ldots, n\}$ consisting of all shuffles of $\{1, \ldots, k\}$ and $\{k+1, \ldots, n\}$. Similar to (5) we see that

$$
\begin{aligned}
& \operatorname{Li}\left(x_{1}, \ldots, x_{k} \mid t_{1}, \ldots, t_{k}\right) \cdot \operatorname{Li}\left(x_{k+1}, \ldots, x_{n} \mid t_{k+1}, \ldots, t_{n}\right)= \\
& \quad \sum_{\sigma \in \Sigma_{k, n-k}} \operatorname{Li}\left(x_{\sigma(1)}, \ldots, x_{\sigma(n)} \mid t_{\sigma(1)}, \ldots, t_{\sigma(n)}\right)+\text { lower depth terms. }
\end{aligned}
$$




\section{Theorem 2.2.}

$$
\begin{array}{r}
I^{*}\left(a_{1}: \ldots: a_{k}: 1 \mid t_{1}, \ldots, t_{k}\right) \cdot I^{*}\left(a_{k+1}: \ldots: a_{n}: 1 \mid t_{k+1}, \ldots, t_{n}\right)= \\
\sum_{\sigma \in \Sigma_{k, n-k}} I^{*}\left(a_{\sigma(1)}, \ldots, a_{\sigma(n)}: 1 \mid t_{\sigma(1)}, \ldots, t_{\sigma(n)}\right) .
\end{array}
$$

Proof. It is not hard to prove the following formula

$$
I^{*}\left[a_{1}: \ldots: a_{m}: 1 \mid t_{1}, \ldots, t_{m}\right]=\int_{0}^{1} \frac{s^{-t_{1}}}{a_{1}-s} d s \circ \ldots \circ \frac{s^{-t_{m}}}{a_{m}-s} d s .
$$

The theorem follows from this and the product formula for iterated integrals.

Here is the simplest case: $I_{1}(x) I_{1}(y)=I_{1,1}(x, y)+I_{1,1}(y, x)$. Indeed,

$$
\int_{0}^{1} \frac{d t}{t-x} \cdot \int_{0}^{1} \frac{d t}{t-y}=\int_{0}^{1} \frac{d t}{t-x} \circ \frac{d t}{t-y}+\int_{0}^{1} \frac{d t}{t-y} \circ \frac{d t}{t-x} .
$$

For multiple $\zeta$ 's these are precisely the relations of Zagier, who conjectured that they provide all the relations between the multiple $\zeta$ 's.

Distribution relations. From the power series expansion we immediately get

Proposition 2.3. If $\left|x_{i}\right|<1$ and $l$ is a positive integer then

$$
\operatorname{Li}\left(x_{1}, \ldots, x_{m} \mid t_{1}, \ldots, t_{m}\right)=\sum_{y_{i}^{l}=x_{i}} \operatorname{Li}\left(y_{1}, \ldots, y_{m} \mid l t_{1}, \ldots, l t_{m}\right) .
$$

\section{The dihedral Lie coalgebra of a commutative group}

1. Definitions. Let $G$ be a commutative group. We will define a bigraded Lie coalgebra $\mathcal{D}_{\bullet \bullet \bullet}(G)=\oplus_{w \geq m \geq 1} \mathcal{D}_{w, m}(G)$. Let us first define a graded abelian group $\hat{\mathcal{D}}_{\bullet}, m(G)$. The group $\mathcal{D}_{\bullet}, m(G)$ is its quotient.

Denote by $C_{m+1}$ the principal homogeneous space of the cyclic group $\mathbb{Z} /(m+$ $1) \mathbb{Z}$. Let $\mathbb{Z}\left[C_{m+1}\right]$ be the abelian group of $\mathbb{Z}$-valued functions on $C_{m+1}$, and $\mathbb{Z}\left[C_{m+1}\right]_{0}$ is its quotient by constants. Let $\operatorname{Pol} \bullet\left(\mathbb{Z}\left[C_{m+1}\right]_{0}\right)$ be the algebra of polynomial functions on $\mathbb{Z}\left[C_{m+1}\right]_{0}$ graded by the degree.

Let $D_{m}$ is the dihedral group of symmetries of the $(m+1)$-gon. Set

$$
G_{0}^{C_{m+1}}:=\quad\left\{g=\left(g_{1}, \ldots, g_{m+1}\right) \in G^{m+1} \mid g_{1} \cdot \ldots \cdot g_{m+1}=1\right\} .
$$

We think about the elements of this group as of $m+1$ elements of $G$ with product 1 located on an oriented circle. Then the group $D_{m}$ acts on (18). Let $\chi_{m}: D_{m} \rightarrow\{ \pm 1\}$ be the character trivial on the cyclic subgroup and sending the involution to $(-1)^{m+1}$. Set

$$
\hat{\mathcal{D}}_{\bullet+m, m}(G):=\left(\mathbb{Z}\left[G_{0}^{C_{m+1}}\right] \otimes_{\mathbb{Z}} \operatorname{Pol} \bullet\left(\mathbb{Z}\left[C_{m+1}\right]_{0}\right) \otimes_{\mathbb{Z}} \chi_{m}\right)_{D_{m}} .
$$


Elements of the group $\hat{\mathcal{D}}_{\bullet, m}(G)$ are presented by the generating functions

$$
\begin{aligned}
& \left\{g_{0}, g_{1}, \ldots, g_{m} \mid t_{0}: \ldots: t_{m}\right\} \quad \text { such that } g_{0} \cdot \ldots \cdot g_{m}=1 \text { and } \\
& \left\{g_{0}, g_{1}, \ldots, g_{m} \mid t_{0}: \ldots: t_{m}\right\}=\left\{g_{0}, g_{1}, \ldots, g_{m} \mid t+t_{0}: \ldots: t+t_{m}\right\}
\end{aligned}
$$

They satisfy the dihedral symmetry relations:

$$
\begin{aligned}
\left\{g_{0}, \ldots, g_{m-1}, g_{m} \mid t_{0}: t_{1}: \ldots: t_{m}\right\} & =\left\{g_{1}, \ldots, g_{m}, g_{0} \mid t_{1}: \ldots: t_{m}: t_{0}\right\} \\
\left\{g_{0}, \ldots, g_{m} \mid t_{0}: \ldots: t_{m}\right\} & =(-1)^{m+1}\left\{g_{m}, \ldots, g_{0} \mid t_{m}: \ldots: t_{0}\right\} .
\end{aligned}
$$

We picture the elements of $\hat{\mathcal{D}}_{\bullet}, m(G)$ as $m+1$ pairs $\left(g_{0}, t_{0}\right), \ldots,\left(g_{m}, t_{m}\right)$ located cyclically on an oriented circle:

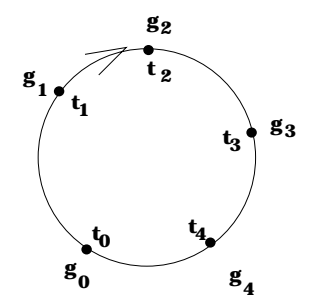

One has $\hat{\mathcal{D}}_{\bullet, m}(G)=\oplus_{w \geq m} \hat{\mathcal{D}}_{w, m}(G)$. We define elements

$$
\left\{g_{1}, \ldots, g_{m}\right\}_{n_{1}, \ldots, n_{m}} \in \hat{\mathcal{D}}_{w, m}(G), \quad w=n_{1}+\ldots+n_{m}, \quad n_{i} \geq 1
$$

generating $\hat{\mathcal{D}}_{w, m}(G)$ as the coefficients of the generating function:

$$
\begin{aligned}
\left\{g_{0}, \ldots, g_{m} \mid t_{0}: \ldots: t_{m}\right\}:= & \\
& \sum_{n_{i}>0}\left\{g_{0}, \ldots, g_{m}\right\}_{n_{1}, \ldots, n_{m}}\left(t_{1}-t_{0}\right)^{n_{1}-1} \ldots\left(t_{m}-t_{0}\right)^{n_{m}-1} .
\end{aligned}
$$

Now one can say that $\hat{\mathcal{D}}_{w, m}(G)$ is generated by the elements (20) satisfying the relations imposed by the dyhedral symmetry.

We call (19) extended nonhomogeneous dihedral words in $G$. One can also parametrize the generators using the extended homogeneous dihedral words:

$$
\begin{gathered}
\left\{g_{0}: g_{1}: \ldots: g_{m} \mid t_{0}, \ldots, t_{m}\right\}, \quad \text { where } t_{0}+\ldots+t_{m}=0, \\
\left\{g \cdot g_{0}: \ldots: g \cdot g_{m} \mid t_{0}, \ldots, t_{m}\right\}=\left\{g_{0}: \ldots: g_{m} \mid t_{0}, \ldots, t_{m}\right\} \quad \text { for any } g \in G,
\end{gathered}
$$

and the dihedral symmetry holds. Namely, the duality between the homogeneous and nonhomogeneous extended dihedral words is given by

$$
\begin{aligned}
&\left\{g_{0}: g_{1}: \ldots: g_{m} \mid t_{0}, \ldots, t_{m}\right\} \longmapsto \\
&\left\{g_{0}^{-1} g_{1}, g_{1}^{-1} g_{2}, \ldots, g_{m}^{-1} g_{0} \mid t_{0}: t_{0}+t_{1}: \ldots: t_{0}+\ldots+t_{m}\right\}, \\
&\left\{g_{0}, g_{1}, \ldots, g_{m} \mid t_{0}: \ldots: t_{m}\right\} \longmapsto \\
&\left\{g_{0}: g_{0} g_{1}: \ldots: g_{0} \ldots g_{m} \mid t_{1}-t_{0}, t_{2}-t_{1}, \ldots, t_{0}-t_{m}\right\} .
\end{aligned}
$$


Definition 3.1. $\mathcal{D}_{\bullet}, m(G)$ is the quotient of $\hat{\mathcal{D}}_{\bullet}, m(G)$ by the following relations:

a) The double shuffle relations $(k+l=m, k \geq 1, l \geq 1)$ :

$$
\begin{aligned}
& \sum_{\sigma \in \Sigma_{k, l}}\left\{g_{0}: g_{\sigma(1)}: \ldots: g_{\sigma(m)} \mid t_{0}, t_{\sigma(1)}, \ldots, t_{\sigma(m)}\right\}=0, \\
& \sum_{\sigma \in \Sigma_{k, l}}\left\{x_{0}, x_{\sigma(1)}, \ldots, x_{\sigma(m)} \mid t_{0}: t_{\sigma(1)}: \ldots: t_{\sigma(m)}\right\}=0
\end{aligned}
$$

b) The distribution relations ( $l \in \mathbb{Z}$ and $|l|$ divides $|G|$ if the group is finite.)

$$
\left\{x_{0}^{l}, x_{1}^{l}, \ldots, x_{m}^{l} \mid t_{0}: t_{1}: \ldots: t_{m}\right\}-\sum_{y_{i}^{l}=x_{i}^{l}}\left\{y_{0}, y_{1}, \ldots, y_{m} \mid l \cdot t_{0}: \ldots: l \cdot t_{m}\right\}=0
$$

except the relation $\{1\}_{1}=\sum_{y^{l}=1}\{y\}_{1}$, which is not supposed to hold.

Example. The distribution relations for $l=-1$ are

$$
\left\{x_{0}^{-1}, x_{1}^{-1}, \ldots, x_{m}^{-1} \mid t_{0}: t_{1}: \ldots: t_{m}\right\}=\left\{x_{0}, x_{1}, \ldots, x_{m} \mid-t_{0}: \ldots:-t_{m}\right\}
$$

Remark. The dihedral symmetry and (21) follow from the double shuffle relations, just copy the proof of theorem 4.1 below.

Let us define a cobracket $\delta: \hat{\mathcal{D}}_{\bullet, \bullet}(G) \longrightarrow \hat{\mathcal{D}}_{\bullet, \bullet}(G) \wedge \hat{\mathcal{D}}_{\bullet, \bullet}(G)$ by setting

$$
\begin{array}{r}
\delta\left\{g_{0}, \ldots, g_{m} \mid t_{0}: \ldots: t_{m}\right\}:= \\
\sum_{i=1}^{m-1} \sum_{j=0}^{m}\left\{g_{j+i+1}, \ldots, g_{j+m}, y_{i j} \mid t_{j+i+1}: \ldots: t_{j+m+1}\right\} \wedge \\
\left\{x_{i j}, g_{j+1}, \ldots, g_{j+i} \mid t_{j}: \ldots: t_{j+i}\right\}
\end{array}
$$

where indices are modulo $m+1$ and $x_{i j} g_{j+1} \ldots g_{j+i}=1, y_{i j} g_{j+i+1} \ldots g_{j+m}=1$. Each term of the formula corresponds to the following procedure: we choose an arc on the circle between the two neighboring distinguished points, and in addition choose a distinguished point different from the ends of the arc. Then we cut the circle in the choosen arc and in the choosen point, make two naturally oriented circles out of it, and then make the extended dihedral word on each of the circles out of the initial word in a natural way.
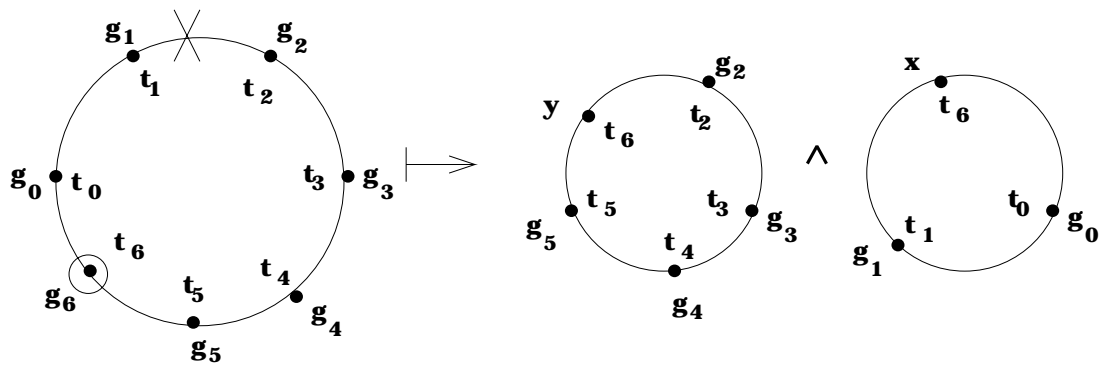
There is a similar formula for the homogeneous dihedral words, just exchange $g$ 's and $t$ 's on the circle. For example

$$
\begin{gathered}
\delta\left\{g_{0}: g_{1}: g_{2} \mid t_{0}, t_{1}, t_{2}\right\}=-\left\{g_{2}: g_{0} \mid t_{0},-t_{0}\right\} \wedge\left\{g_{1}: g_{2} \mid t_{1},-t_{1}\right\}- \\
-\left\{g_{0}: g_{1} \mid t_{1},-t_{1}\right\} \wedge\left\{g_{2}: g_{0} \mid t_{2},-t_{2}\right\}-\left\{g_{1}: g_{2} \mid t_{2},-t_{2}\right\} \wedge\left\{g_{0}: g_{1} \mid t_{0},-t_{0}\right\} .
\end{gathered}
$$

Theorem 3.2. $\delta$ provides the structure of bigraded Lie coalgebra on both $\hat{\mathcal{D}}_{\bullet, \bullet}(G)$ and $\mathcal{D}_{\bullet, \bullet}(G)$.

3.1. 2. The cyclotomic Lie algebra and mixed Tate motives over $S_{N}$. Recall that a mixed Hodge structure is called a Hodge-Tate structure if all the Hodge numbers $h^{p, q}$ with $p \neq q$ are zero. The category of mixed $\mathbb{Q}$-HodgeTate structures is canonically equivalent to the category of finite dimensional comodules over a certain graded pro-Lie coalgebra $\mathcal{L}_{\bullet}^{H T}$ over $\mathbb{Q}$ (see [BGSV], [G4]). One can attach to the iterated integral related to $L i_{n_{1}, \ldots, n_{m}}\left(x_{1}, \ldots, x_{m}\right)$ by theorem 2.1 above an element $L i_{n_{1}, \ldots, n_{m}}^{\mathcal{H}}\left(x_{1}, \ldots, x_{m}\right) \in \mathcal{L}_{w}^{H T}$, the motivic multiple polylogarithm. See s.9, 11 of [G2] (or [G1]) where $\mathcal{L}_{\bullet}^{H T}$ and a $w$-framed mixed Hodge-Tate structure $L i_{n_{1}, \ldots, n_{m}}^{\mathcal{H}}\left(x_{1}, \ldots, x_{m}\right)$ were defined. The framed mixed Hodge structures corresponding to the divergent numbers (4) are defined as the limiting Hodge structures, (compare with [G1] and s.7). For instance $L i_{1}^{\mathcal{H}}(1)=0$.

Definition 3.3. $\mathcal{C}_{w}(N)$ is the $\mathbb{Q}$-subspace of $\mathcal{L}_{w}^{H T}$ generated by the motivic multiple polylogarithms at $N$-th roots of unity of weight $w$.

Theorem 3.4. $\mathcal{C}_{\bullet}(N):=\oplus_{w \geq 1} \mathcal{C}_{w}(N)$ is a Lie subcoalgebra in $\mathcal{L}_{\bullet}^{H T}$.

We define the cyclotomic Lie algebra $C_{\bullet}(N)$ as the (graded) dual of $\mathcal{C} \bullet(N)$.

The hypothetical abelian category of mixed Tate motives over the scheme $S_{N}$ is supposed to be canonically equivalent to the category of finite dimensional modules over a graded Lie algebra $L\left(S_{N}\right)$ e, called the motivic Lie algebra of that scheme, (see [G4] for details). $L\left(S_{N}\right)$. is isomorphic to a free graded Lie algebra generated by the $\mathbb{Q}$-spaces $K_{2 n-1}\left(S_{N}\right) \otimes \mathbb{Q}$ in degree $n, n \geq 1$.

One can prove that the Lie algebra $C_{\bullet}(N)$ has the same generators, so it is

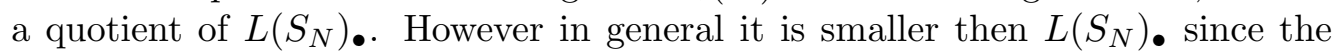
main result of [G3] implies that $C \bullet(N)$ is not free when $N$ is sufficiently big. For instance, if $N=p$ is a prime then

$$
H_{(2)}^{2}(C \bullet(p), \mathbb{Q})=H^{1}\left(X_{1}(p), \mathbb{Q}\right)_{+} \oplus \mathbb{Z}\left[\zeta_{p}\right]^{*} \otimes \mathbb{Q}
$$

where $X_{1}(p)$ is the modular curve and + means the coinvariants of the complex conjugation. So it is non zero if $p>3$.

The relation between the dihedral and cyclotomic Lie algberas. Let $\mathcal{C}_{\bullet}, \bullet(N)$ be the associate graded with respect to the depth filtration on the Lie coalgebra $\mathcal{C}_{\bullet}(N)$. 
Theorem 3.5. Assume that $x_{i}^{N}=1$. Then the map

$$
\left\{x_{1}, \ldots, x_{m}\right\}_{n_{1}, \ldots, n_{m}} \longmapsto L i_{n_{1}, \ldots, n_{m}}^{\mathcal{H}}\left(x_{1}, \ldots, x_{m}\right)
$$

provides a surjective morphism of bigraded Lie coalgebras $\mathcal{D} \bullet, \bullet\left(\mu_{N}\right) \rightarrow \mathcal{C} \bullet, \bullet(N)$

The subspace $\mathcal{D}_{1,1}(1)$ is generated by $\{1\}_{1}$. Notice that $L i_{1}^{\mathcal{H}}(1)=0$.

Conjecture 3.6. $\mathcal{D}_{\bullet, \bullet}(1) / \mathcal{D}_{1,1}(1)=\mathcal{C}_{\bullet, \bullet}(1)$.

But already $\mathcal{C}_{1,1}(N)$ is smaller then $\mathcal{D}_{1,1}(N)$ if $N$ is not prime ( take $N=25$ ). Let $(l, N)=1$. One has canonical homomorphism

$$
\varphi_{N}^{l}: \operatorname{Gal}(\overline{\mathbb{Q}} / \mathbb{Q}) \longrightarrow \operatorname{Out\pi }_{1}^{(l)}\left(\mathbb{P}^{1} \backslash\left\{0,\left\{\zeta_{N}^{\alpha}\right\}, \infty\right\}\right) .
$$

The motivic philosophy suggests that the Lie algebra of the Zariski closure of the image of $\varphi_{N}^{l}$ is isomorphic to the Lie algebra $C \cdot(N)^{\vee} \otimes_{\mathbb{Q}} \mathbb{Q}_{l}$.

\section{The modular complex}

Let $L_{m}$ be a lattice of rank $m$. The rank $m$ modular complex is a complex of left $G L_{m}(\mathbb{Z})$-modules in degrees $[1, m]$, denoted $M_{(m)}^{\bullet}\left(L_{m}\right)$ or simply $M_{(m)}^{\bullet}$ :

$$
M_{(m)}^{1} \stackrel{\partial}{\longrightarrow} M_{(m)}^{2} \stackrel{\partial}{\longrightarrow} \ldots \stackrel{\partial}{\longrightarrow} M_{(m)}^{m}
$$

1. The groups $M_{(m)}^{l}$. Let $X$ be a set and $\mathbb{Z}[[X]]$ be the abelian group of infinite $\mathbb{Z}$-linear combinations $\sum n_{x}\{x\}$ of the generators $\{x\}$, where $x \in X$. The set $\mathcal{P}_{m}$ of basises $\left(v_{1}, \ldots, v_{m}\right)$ of $L_{m}$ is a principal homogeneous space of $G L_{m}(\mathbb{Z})$. Then $M_{(m)}^{l}=\mathbb{Z}\left[\left[\mathcal{P}_{m}\right]\right] / R_{(m)}^{l}$. The "relations" $R_{(m)}^{l}$ are defined below.

By definition the generators of the group $M_{(m)}^{1}$ are

$$
\left[v_{1}, \ldots, v_{m}\right], \quad \text { where }\left(v_{1}, \ldots, v_{m}\right) \text { is a basis of } L_{m}
$$

We will use other two notation for them:

$$
\begin{gathered}
\left\langle v_{0}, \ldots, v_{m}\right\rangle:=\left[v_{1}, \ldots, v_{m}\right] \quad \text { where } v_{0}+\ldots+v_{m}=0, \quad \text { and } \\
{\left[v_{1}: \ldots: v_{m}\right]:=\left[v_{2}-v_{1}, v_{3}-v_{2}, \ldots, v_{m}-v_{m-1},-v_{m}\right]}
\end{gathered}
$$

Notice that if $v_{1}, \ldots, v_{m}$ is a basis of $L_{m}$ and $v_{0}+\ldots+v_{m}=0$, then omitting any vector of $v_{0}, \ldots, v_{m}$ we also get a basis.

The relations are given by the double shuffle relations: for any $1 \leq k \leq m-1$

$$
\begin{aligned}
s\left(v_{1}, \ldots, v_{k} \mid v_{k+1}, \ldots, v_{m}\right) & :=\sum_{\sigma \in \Sigma_{k, m-k}}\left[v_{\sigma(1)}, \ldots, v_{\sigma(m)}\right]=0 \\
s\left(v_{1}: \ldots: v_{k} \mid v_{k+1}: \ldots: v_{m}\right):= & \sum_{\sigma \in \Sigma_{k, m-k}}\left[v_{\sigma(1)}: \ldots: v_{\sigma(m)}\right]=0
\end{aligned}
$$


Theorem 4.1. The double shuffle relations imply the following dihedral symmetry relations: $\left[v_{1}, \ldots, v_{m}\right]=\left[-v_{1}, \ldots,-v_{m}\right]$, and $<v_{0}, \ldots, v_{m}>=<v_{1}, \ldots, v_{m}, v_{0}>,<v_{0}, \ldots, v_{m}>=(-1)^{m+1}<v_{m}, \ldots, v_{0}>$ Proof. Starting from the shuffle relation $s\left(v_{1} \mid v_{2}: \ldots: v_{m}\right)$ :

$$
0=\left[v_{1}: v_{1}+v_{2}: \ldots: v_{1}+\ldots+v_{m}\right]+\ldots+\left[v_{2}: v_{2}+v_{3}: \ldots: v_{1}+\ldots+v_{m}\right]
$$

we rewrite it as $\left[v_{2}, \ldots, v_{m}, v_{0}\right]+s\left(v_{1} \mid v_{3}, \ldots, v_{m}, v_{0}\right)-\left[v_{3}, \ldots, v_{m}, v_{0}, v_{1}\right]=0$, getting the cyclic symmetry for the generators $\left[v_{1}, \ldots, v_{m}\right]$. It is easy see that

$$
\sum_{k=0}^{m}(-1)^{k} s\left(v_{k}: v_{k-1}: \ldots: v_{1} \mid v_{k+1}: \ldots: v_{m}\right)=0
$$

which means that $\left[v_{1}: v_{2}: \ldots: v_{m-1}: v_{m}\right]=(-1)^{m+1}\left[v_{m}: v_{m-1}: \ldots: v_{2}\right.$ : $\left.v_{1}\right]$. A similar result is proved for the $\left[v_{1}, \ldots, v_{m}\right]$ generators. Using this we get

$$
\begin{gathered}
{\left[-v_{1},-v_{2}, \ldots,-v_{m}\right]=\left[v_{1}+\ldots+v_{m}: \ldots: v_{m-1}+v_{m}: v_{m}\right]=} \\
(-1)^{m+1}\left[v_{m}: v_{m-1}+v_{m}: \ldots: v_{1}+\ldots+v_{m}\right]= \\
(-1)^{m+1}\left[v_{m-1}, \ldots, v_{1}, v_{0}\right]=\left[v_{0}, \ldots, v_{m-1}\right] .
\end{gathered}
$$

So we proved the dihedral symmetry for the generators $\left[v_{1}, \ldots, v_{m}\right]$, and thus for the generators $\left[v_{1}: \ldots: v_{m}\right]$.

The generators of the group $M_{(m)}^{l}$ are the symbols

$$
\left[v_{1}, \ldots, v_{m_{1}}\right] \wedge \ldots \wedge\left[v_{m_{l-1}+1}, \ldots, v_{m_{l}}\right]
$$

where $m=k_{1}+\ldots+k_{l}, m_{i}:=k_{1}+\ldots+k_{i}, \quad\left(v_{1}, \ldots, v_{m}\right)$ is a basis of $L_{m}$, each of the blocks $\left[A_{i}\right]:=\left[v_{m_{i}+1}, \ldots, v_{m_{i+1}}\right]$ satisfy the double shuffle relations, and the blocks $\left[A_{i}\right]$ anticommute:

$$
\ldots \wedge\left[A_{i}\right] \wedge\left[A_{i+1}\right] \wedge \ldots=-\ldots \wedge\left[A_{i+1}\right] \wedge\left[A_{i}\right] \wedge \ldots
$$

2. The differential $\partial$. We define a differential $\partial: M_{(m)}^{1} \longrightarrow M_{(m)}^{2}$ by

$$
\partial:\left\langle v_{1}, \ldots, v_{m+1}>\longmapsto-\operatorname{Cycle}_{m+1}\left(\sum_{k=1}^{m-1}\left[v_{1}, \ldots, v_{k}\right] \wedge\left[v_{k+1}, \ldots, v_{m}\right]\right)\right.
$$

where $\operatorname{Cycle}_{m+1}\left(f\left(v_{1}, \ldots, v_{m}\right)\right):=\sum_{i=1}^{m+1} f\left(v_{i}, \ldots, v_{i+m}\right)$, the indices are modulo $m+1$. Then we extend $\partial$ to the complex $M_{(m)}^{\bullet}$ using the Leibniz rule:

$$
\partial\left(\left[A_{1}\right] \wedge\left[A_{2}\right] \wedge \ldots \wedge\left[A_{n}\right]\right):=\sum_{i=1}^{n}(-1)^{i}\left[A_{1}\right] \wedge \ldots \wedge \partial\left[A_{i}\right] \wedge \ldots\left[A_{n}\right]
$$

Theorem 4.2. The differential $\partial$ is a well defined homomorphism of abelian groups. One has $\partial^{2}=0$. 
Remark. The modular complex is not the standard cochain complex of any graded Lie coalgebra.

3. Modular complexes for $G L_{2}$ and $G L_{3}$. Here is explicit description of the modular complexes $M_{(m)}^{\bullet}$ for $m=1,2,3$.

1. $m=1$. Then $[v]=[-v]$, and $M_{(1)}^{1}=\mathbb{Z}$.

2. $m=2$. Then the modular complex is $M_{(2)}^{1} \stackrel{\partial}{\longrightarrow} M_{(2)}^{2}$. The group $M_{(2)}^{1}$ is generated by $\left\langle v_{0}, v_{1}, v_{2}>\right.$ where $v_{0}+v_{1}+v_{2}=0$ and $\left(v_{1}, v_{2}\right)$ is a basis in $L_{2}$. The differential is:

$$
\partial:\left\langle v_{0}, v_{1}, v_{2}>\longmapsto-\left[v_{1}\right] \wedge\left[v_{2}\right]-\left[v_{2}\right] \wedge\left[v_{0}\right]-\left[v_{0}\right] \wedge\left[v_{1}\right]\right.
$$

3. $m=3$. The complex looks as follows: $M_{(3)}^{1} \stackrel{\partial}{\longrightarrow} M_{(3)}^{2} \stackrel{\partial}{\longrightarrow} M_{(3)}^{3}$. The differentials are

$$
\begin{gathered}
\partial:<v_{0}, v_{1}, v_{2}, v_{3}> \\
-\left[v_{1}, v_{2}\right] \wedge\left[v_{3}\right]-\left[v_{2}, v_{3}\right] \wedge\left[v_{0}\right]-\left[v_{3}, v_{0}\right] \wedge\left[v_{1}\right]-\left[v_{0}, v_{1}\right] \wedge\left[v_{2}\right] \\
-\left[v_{0}\right] \wedge\left[v_{1}, v_{2}\right]-\left[v_{1}\right] \wedge\left[v_{2}, v_{3}\right]-\left[v_{2}\right] \wedge\left[v_{3}, v_{0}\right]-\left[v_{3}\right] \wedge\left[v_{0}, v_{1}\right] \\
\partial:\left[v_{1}, v_{2}\right] \wedge\left[v_{3}\right] \quad \longmapsto-\left(\left[v_{1}\right] \wedge\left[v_{2}\right]+\left[v_{2}\right] \wedge\left[-v_{1}-v_{2}\right]+\left[-v_{1}-v_{2}\right] \wedge\left[v_{1}\right]\right) \wedge\left[v_{3}\right] .
\end{gathered}
$$

4. Modular complexes and modular cohomology. We define the modular complex $M C^{*}(\Gamma, V)$ of a subgroup $\Gamma$ of $G L_{m}(\mathbb{Z})$ with coefficients in a right $G L_{m}$-module $V$ as follows:

$$
M C^{*}(\Gamma, V):=\quad V \otimes_{\Gamma} M_{(m)}^{*} .
$$

Its cohomology are called the modular cohomology $M H^{*}(\Gamma, V)$ of $\Gamma$ with coefficients in $V$. If $E$ is the one element group, then $M C^{*}(E, \mathbb{Z})=M_{(m)}^{*}$.

The group $G L_{m}(\mathbb{Z})$ acts from the right on $\mathbb{Z}\left[\Gamma \backslash G L_{m}(\mathbb{Z})\right]$, and thus on $\mathbb{Z}\left[\Gamma \backslash G L_{m}(\mathbb{Z})\right] \otimes_{\mathbb{Z}} V$. It is easy to see (Shapiro's lemma) that one has

$$
M C^{*}(\Gamma, V)=\left(\mathbb{Z}\left[\Gamma \backslash G L_{m}(\mathbb{Z})\right] \otimes_{\mathbb{Z}} V\right) \otimes_{G L_{m}(\mathbb{Z})} M_{(m)}^{*} .
$$

5. A map from the modular complex for $\Gamma_{1}(N ; m)$ with coefficients in $\mathbb{Z}\left[t_{1}, \ldots, t_{m}\right]$ to the cochain complex of the dihedral Lie coalgebra of $\mu_{N}$. One has

$$
\begin{aligned}
\Gamma_{1}(N ; m) \backslash G L_{m}(\mathbb{Z})=\left\{\left(\alpha_{1}, \ldots, \alpha_{m}\right) \mid\right. \\
\left.\alpha_{i} \in \mathbb{Z} / N \mathbb{Z}, \quad \text { g.c.d. }\left(\alpha_{1}, \ldots, \alpha_{m}, N\right)=1\right\} .
\end{aligned}
$$

Indeed, the group $G L_{m}(\mathbb{Z})$ acts from the right on $(\mathbb{Z} / N \mathbb{Z})^{m}$ and $\Gamma_{1}(N ; m)$ is the stabilizer of the element $(0, \ldots, 0,1)$. The $G L_{m}(\mathbb{Z})$-orbit of this element is the right hand side of (28). 
Consider the right $G L_{m}$-module structure on $\mathbb{Z}\left[t_{1}, \ldots, t_{m}\right]$ given by $t_{i} \cdot g:=$ $\sum_{j=1}^{m}\left(g^{-1}\right)_{i j} t_{j}$. We will construct a canonical morphism of complexes

$$
\mu(N)_{\bullet, m}^{*}: M C^{*}\left(\Gamma_{1}(N ; m), \mathbb{Z}\left[t_{1}, \ldots, t_{m}\right]\right) \quad \longrightarrow \quad \Lambda^{*}\left(\mathcal{D}_{\bullet, \bullet}\left(\mu_{N}\right)\right)_{\operatorname{depth}}=\mathrm{m} .
$$

where the left hand side is graded by (degree of a polynomial in $\left.t_{i}\right)+m$, and the right hand side by the weight.

Let $S^{k}\left[t_{1}, \ldots, t_{m}\right]$ be the abelian group of degree $k$ polynomials in $t_{1}, \ldots, t_{m}$ with integer coefficients. We will use (27). Let us define first maps

$$
\mu_{w, m}^{1}: M C^{1}\left(\Gamma_{1}(N ; m), S^{w-m}\left[t_{1}, \ldots, t_{m}\right]\right) \quad \longrightarrow \quad \mathcal{D}_{m, w}\left(\mu_{N}\right) .
$$

Choose a basis $\left(v_{1}, \ldots, v_{m}\right)$ in $V_{m}$. Let $\alpha_{0}+\alpha_{1}+\ldots+\alpha_{m}=0$. Set

$$
\begin{gathered}
\mu(N)_{\bullet, m}^{1}: \quad \sum_{n_{i}>0}\left(\alpha_{1}, \ldots, \alpha_{m}\right) \otimes t_{1}^{n_{1}-1} \ldots t_{m}^{n_{m}-1} \otimes\left[v_{1}, \ldots, v_{m}\right] \longmapsto \\
\left\{\zeta_{N}^{\alpha_{0}}, \zeta_{N}^{\alpha_{1}}, \ldots, \zeta_{N}^{\alpha_{m}} \mid 0: t_{1}: \ldots: t_{m}\right\}:= \\
\sum_{n_{i}>0}\left\{\zeta_{N}^{\alpha_{0}}, \zeta_{N}^{\alpha_{1}}, \ldots, \zeta_{N}^{\alpha_{m}}\right\}_{n_{1}, \ldots, n_{m}} t_{1}^{n_{1}-1} \ldots t_{m}^{n_{m}-1} .
\end{gathered}
$$

Lemma 4.3. The map $\mu_{w, m}^{1} \otimes \mathbb{Q}$ is a surjective homomorphism.

Proof. It is well defined thanks to the definitions of the modular complex and the dihedral Lie coalgebra. It is surjective because of the distribution relations.

Define a map

$$
\mu(N)_{\bullet, m}^{l}: M C^{l}\left(\Gamma_{1}(N ; m), \mathbb{Z}\left[t_{1}, \ldots, t_{m}\right]\right) \quad \longrightarrow \quad \mathcal{D}_{m, \bullet}\left(\mu_{N}\right)
$$

as follows. Choose a primitive $N$-th root of unity $\zeta_{N}$. Then

$$
\begin{aligned}
\mu(N)_{w, m}^{l}: \sum_{n_{i}>0}\left(\alpha_{1}, \ldots, \alpha_{m}\right) \otimes t_{1}^{n_{1}-1} \ldots t_{m}^{n_{m}-1} \otimes\left[v_{1}, \ldots, v_{n_{1}}\right] \wedge \ldots \wedge \\
{\left[v_{n_{l-1}+1}, \ldots, v_{n_{l}}\right] \longmapsto\left\{\zeta_{N}^{\beta_{1}}, \zeta_{N}^{\alpha_{1}}, \ldots, \zeta_{N}^{\alpha_{n_{1}}} \mid 0: t_{1}: \ldots: t_{n_{1}}\right\} \wedge \ldots \wedge } \\
\left\{\zeta_{N}^{\beta_{l}}, \zeta_{N}^{\alpha_{n_{l-1}+1}}, \ldots, \zeta_{N}^{\alpha_{n_{l}}} \mid 0: t_{n_{l-1}+1}: \ldots: t_{n_{l}}\right\}
\end{aligned}
$$

where by definition $\beta_{i}+\alpha_{n_{i-1}+1}+\ldots+\alpha_{n_{i}}=0$ for $i=1, \ldots, l$.

Proof of theorem 1.2. By the very definitions the map $\mu(N)_{\bullet}^{*} \bullet$ is a well defined morphism of complexes. It is surjective thanks to lemma 4.3. If $N=1$ we have the distribution relations only for $l=-1$, and they follow from the double shuffle relations by theorem 4.1 . So the map $\mu(1)_{\bullet, \bullet}^{*}$ is an isomorphism.

If $N$ is a prime and $w=m>1$ there is one additional distribution relation, $\{1, \ldots, 1\}_{1, \ldots, 1}=\sum_{x_{i}^{p}=1}\left\{x_{1}, \ldots, x_{m}\right\}_{1, \ldots, 1}$. But the shuffle relations give $\sum_{\sigma \in S_{m}}\left\{x_{\sigma(1)}, \ldots, x_{\sigma(m)}\right\}_{1, \ldots, 1}=0$, which imply the distribution relation. 


\section{The Voronoi complex}

1. Voronoi's cell decomposition of $S L_{n}(\mathbb{R}) / S O_{n}$. Let $Q\left(V_{m}\right)$ be the space of quadratic forms in an $m$-dimensional vector space $V_{m}$ over $\mathbb{R}$. Denote by $\mathcal{P}\left(V_{m}\right)$ (resp. $\left.\overline{\mathcal{P}}\left(V_{m}\right)\right)$ the cone of positive definite (resp. non negative definite) quadratic forms in $V_{m}$. Then

$$
\mathbb{H}_{m}:=S L_{m}(\mathbb{R}) / S O(m)=\mathcal{P}\left(V_{m}\right) / \mathbb{R}_{+}^{*}
$$

and $\overline{\mathcal{P}}\left(V_{m}\right) / \mathbb{R}_{+}^{*}$ is its compactification. For example $\mathbb{H}_{2}$ is the hyperbolic plane.

Any vector $f \in V_{m}^{*}$ defines a degenerate non negatively definite quadratic form $\varphi(f):=(f, x)^{2}$. Choose a lattice $L_{m} \subset V_{m}^{*}$. Let $G L\left(L_{m}\right) \subset G L\left(V_{m}\right)$ be the subgroup preserving the lattice $L_{m}$. Take the convex hull $\mathcal{C}\left(L_{m}\right)$ of the vectors $\varphi(l)$ in the cone $\overline{\mathcal{P}}\left(V_{m}\right)$ when $l$ runs through all non zero primitive vectors of the lattice $L_{m}$. It is of codimension 1 in $Q\left(V_{m}\right)$ and has a structure of an infinite polyhedra. Its faces are certain convex polyhedras with vertices $\varphi\left(l_{1}\right), \ldots, \varphi\left(l_{n}\right)$, $l_{i} \in L_{m}$. Projecting it onto $\mathcal{P}\left(V_{m}\right) / \mathbb{R}_{+}^{*}$ we get a $G L\left(L_{m}\right)$-invariant polyhedral decomposition of $\mathbb{H}_{m}$ called Voronoi's cell decomposition. Set

$\varphi\left(l_{1}, \ldots, l_{n}\right):=\left\{\lambda_{1} \cdot \varphi\left(l_{1}\right)+\ldots+\lambda_{n} \cdot \varphi\left(l_{n}\right)\right\} / \mathbb{R}^{*}, \quad \lambda_{i} \geq 0, \quad \lambda_{1}+\ldots+\lambda_{n}=1$.

The cells of the projection of $\mathcal{C}\left(L_{m}\right)$ are polyhedras $\varphi\left(l_{1}, \ldots, l_{n}\right)$ for certain vectors $l_{1}, \ldots, l_{n} \in L_{m}$. They satisfy the condition $\mathrm{rk}\left\langle l_{1}, \ldots, l_{n}\right\rangle=m$.

The non zero vectors of the lattice $L_{m}$ minimizing the values of a form $F$ on $V_{m}^{*}$ on $L_{m} \backslash 0$ are called the minimal vectors of $F$. A quadratic form $F$ in $V_{m}^{*}$ is called perfect if the number of minimal vectors of $F$ is at least $\frac{m(m+1)}{2}=\operatorname{dim} Q\left(V_{m}^{*}\right)$.

Let $s$ be a codimension 1 face of $\mathcal{C}\left(L_{m}\right)$. Let $h(s)$ be the codimension 1 subspace in $Q\left(V_{m}\right)$ parallel to the face $s$.

Voronoi's lemma. $F \in Q\left(V_{m}^{*}\right)$ is orthogonal to the subspace $h(s)$ if and only if $F$ is a perfect quadratic form. In this case $\left\{ \pm l_{1}, \ldots, \pm l_{n}\right\}$ is the set of minimal vectors for $F$.

Proof. One has $(F, \varphi(l))=F(l)$. Let $(F, x)=c$ be the equation of the hyperplane $h(s)$. Since $\mathcal{C}\left(L_{m}\right)$ is a convex hull it is located in just one of the subspaces $(F, x)<c$ or $(F, x)>c$. Since $(F, \varphi(l))=F(l)$ could be arbitrary big, the domain $\{x \mid(F, x)<c\}$ does not intersect $\mathcal{C}\left(L_{m}\right)$. Further, $(F, \varphi(l))=c$ for any vertex $\varphi(l)$ of the face $\varphi$, so such $l$ 's are minimal vectors for $F$. Since the face $\varphi$ is of codimension 1 , the number of its vertices is at least $\operatorname{dim} Q\left(V_{m}\right)$. So the form $F$ is perfect. The lemma is proved.

Let $v_{1}, \ldots, v_{m+1}$ vectors of $L_{m}$ such that $v_{1}+\ldots+v_{m+1}=0$ and $v_{1}, \ldots, v_{m}$ is a basis of $L$. Set

$$
v_{i, j}:=v_{i}+v_{i+1}+\ldots+v_{j-1}+v_{j}, \quad 1 \leq i, j \leq m+1, \quad i \neq j-1
$$

and indices are modulo $m+1$. The configuration of vectors $v_{i, j}$ in (29) is linearly equivalent to the configuration of the roots of the root system $A_{m}$. See fig. 4 for the configuration of points in $P^{2}$ corresponding to the root system $A_{2}$. 
The convex hull of $\varphi\left(v_{i, j}\right)$ is a Voronoi cell, called the cell of type $A_{m}$, and the correseponding perfect form is the quadratic form of the root system $A_{m}$ with the the set of mimimal vectors given by the roots.

Voronoi's theorem. [V], [M] For $m=2,3$ any cell of top dimension in the Voronoi decomposition of $\mathbb{H}_{m}$ is $G L_{m}\left(L_{m}\right)$-equivalent to a cell of type $A_{m}$.

2. The Voronoi complex. Let

$$
\left(V_{\bullet}^{(m)}, d\right)=\left(V_{\bullet}\left(L_{m}\right), d\right):=\quad V_{\frac{m(m+1)}{2}-1}^{(m)} \stackrel{d}{\longrightarrow} V_{\frac{m(m+1)}{2}-2}^{(m)} \stackrel{d}{\longrightarrow} \ldots \stackrel{d}{\longrightarrow} V_{m-1}^{(m)}
$$

be the complex of (infinite) chains with closed supports associated with the Voronoi decomposition of $\mathbb{H}_{m}$. We call it the Voronoi complex of the lattice $L_{m}$. An isomorphism between lattices lifts to an isomorphism between the corresponding Voronoi complexes, justifying name the Voronoi complex for $G L_{m}$.

\section{Relating the modular and Voronoi complexes for $G L_{2}$ and $G L_{3}$}

The modular complex $M_{(m)}^{\bullet}$ is a cohomological complex placed in degrees $[1, m+1]$. Let us cook up out of him a homological complex sitting in degrees $[2 m, m]$ by setting $M_{\bullet}^{(m)}:=M_{(m)}^{2 m+1-\bullet}$.

1. An isomorphism between the modular and Voronoi complexes for $G L_{2}$. The Voronoi complex for $G L_{2}$ looks as follows: $V_{\bullet}^{(2)}:=V_{2}^{(2)} \stackrel{d}{\longrightarrow} V_{1}^{(2)}$. It is the chain complex of the classical modular triangulation of the hyperbolic plane, see the figure on page 2

Define a map of $G L_{2}(\mathbb{Z})$-modules $\psi^{(2)}: M_{\bullet}^{(2)} \longrightarrow V_{\bullet}^{(2)}$ as follows. Let $v_{1}, v_{2}$ be a basis of $L_{2}, v_{1}+v_{2}+v_{3}=0$. Set

$$
\left[v_{1}, v_{2}\right] \longmapsto \varphi\left(v_{1}, v_{2}, v_{3}\right), \quad\left[v_{1}\right] \wedge\left[v_{2}\right] \longmapsto \varphi\left(v_{1}, v_{2}\right) .
$$

Theorem 6.1. a) The map $\psi^{(2)}$ is an isomorphism of complexes $M_{\bullet}^{(2)} \longrightarrow V_{\bullet}^{(2)}$.

b) Let $\Gamma$ be a subgroup of $G L_{2}(\mathbb{Z})$. Then for any $G L_{2}$-module $V$ there are canonical isomorphisms $H_{M_{(2)}^{i}}(\Gamma, V) \otimes \mathbb{Q}=H^{i-1}(\Gamma, V) \otimes \mathbb{Q}$.

Proof. a) When $\left(v_{1}, v_{2}\right)$ run through all basises of the lattice $L_{2}$, the triangles $\varphi\left(v_{1}, v_{2}, v_{3}\right)$ where $v_{1}+v_{2}+v_{3}=0$ are cells of type $A_{2}$, and so by Voronoi's theorem produce all the 2-cells of Voronoi's complex for $G L_{2}$. Since $\psi^{(2)}$ commutes with the differentials, we obviously get an isomorphism of complexes.

b) Voronoi's complex $V_{\bullet}^{(2)}$ is a resolution of the trivial $G L_{2}(\mathbb{Z})$-module $\mathbb{Z}[2]$. This resolution is free over a certain finite index subgroup $\Gamma \subset G L_{2}(\mathbb{Z})$. 


\section{A quasiisomorphism between the modular and truncated Voronoi} complexes for $G L_{3}$. The Voronoi complex for $G L_{3}$ looks as follows:

$$
\left(V_{\bullet}^{(3)}, d\right):=V_{5}^{(3)} \stackrel{d}{\longrightarrow} V_{4}^{(3)} \stackrel{d}{\longrightarrow} V_{3}^{(3)} \stackrel{d}{\longrightarrow} V_{2}^{(3)}
$$

We will suppose that $v_{1}, v_{2}, v_{3}$ is a basis in $L_{3}$ and $v_{1}+v_{2}+v_{3}+v_{4}=0, \quad v_{12}:=v_{1}+v_{2}, \quad v_{23}:=v_{2}+v_{3}, \quad v_{13}:=v_{1}+v_{3}, \quad \ldots$

By Voronoi's theorem the $G L_{3}(\mathbb{Z})$-orbits of the 5 -symplex $\varphi\left(v_{1}, v_{2}, v_{3}\right.$, $\left.v_{4}, v_{12}, v_{23}\right)$ and its faces provide all cells of the Voronoi decomposition for $G L_{3}$.

Define a map $\psi^{(3)}: M_{\bullet}^{(3)} \longrightarrow V_{\bullet}^{3} / d V_{5}^{(3)}$ as follows:

$$
\begin{gathered}
{\left[v_{1}\right] \wedge\left[v_{2}\right] \wedge\left[v_{3}\right] \longmapsto \varphi\left(v_{1}, v_{2}, v_{3}\right)} \\
{\left[v_{1}, v_{2}\right] \wedge\left[v_{3}\right] \longmapsto \varphi\left(v_{1}, v_{2},-v_{1}-v_{2}, v_{3}\right),} \\
{\left[v_{1}, v_{2}, v_{3}\right] \longmapsto \varphi\left(v_{1}, v_{2}, v_{3}, v_{4}, v_{12}\right)-\varphi\left(v_{1}, v_{2}, v_{3}, v_{4}, v_{23}\right) .}
\end{gathered}
$$

Theorem 6.2. a) The map $\psi^{(3)}$ provides an injective morphism of complexes of $G L_{3}(\mathbb{Z})$-modules $M_{\bullet}^{(3)} \longrightarrow V_{\bullet}^{(3)} / d V_{5}^{(3)}$. It is a quasiisomorphisms.

b) Let $\Gamma$ be a subgroup of $G L_{3}(\mathbb{Z})$. Then for any $G L_{3}$-module $V$ there are canonical isomorphisms $H M_{(3)}^{i}(\Gamma, V) \otimes \mathbb{Q}=H^{i}(\Gamma, V) \otimes \mathbb{Q}, \quad i \geq 1$.

Proof. b) => a). It is similar to the proof of theorem $6.1 \mathrm{a}$ ).

a) Let us show that $\psi^{(3)}$ is a well defined morphism of complexes. The map $\psi^{(3)}$ sends the first shuffle relation to zero already in the group $V_{4}^{(3)}$ :

$$
\begin{aligned}
\psi^{(3)}: & s\left(v_{1} \mid v_{2}, v_{3}\right)= \\
& <v_{1}, v_{2}, v_{3}, v_{4}>+<v_{2}, v_{1}, v_{3}, v_{4}>+<v_{2}, v_{3}, v_{1}, v_{4}>\longmapsto \\
\varphi & \left(v_{1}, v_{2}, v_{3}, v_{4}, v_{12}\right)-\varphi\left(v_{1}, v_{2}, v_{3}, v_{4}, v_{23}\right)+\varphi\left(v_{2}, v_{1}, v_{3}, v_{4}, v_{12}\right)- \\
- & \varphi\left(v_{2}, v_{1}, v_{3}, v_{4}, v_{13}\right)+\varphi\left(v_{2}, v_{3}, v_{1}, v_{4}, v_{23}\right)-\varphi\left(v_{2}, v_{3}, v_{1}, v_{4}, v_{13}\right)=0 .
\end{aligned}
$$

The second shuffle relation looks as follows:

$$
\begin{aligned}
& s\left(u_{1} \mid u_{2}: u_{3}\right):=\left[u_{1}: u_{2}: u_{3}\right]+\left[u_{2}: u_{1}: u_{3}\right]+\left[u_{2}: u_{3}: u_{1}\right]= \\
& \quad\left[u_{2}-u_{1}, u_{3}-u_{2},-u_{3}\right]+\left[u_{1}-u_{2}, u_{3}-u_{1},-u_{3}\right]+\left[u_{3}-u_{2}, u_{1}-u_{3},-u_{1}\right] .
\end{aligned}
$$

Changing the variables $v_{1}:=u_{2}-u_{1}, v_{2}:=u_{3}-u_{2}, v_{3}:=-u_{3}$ we get

$$
<v_{1}, v_{2}, v_{3}, v_{4}>+<-v_{1}, v_{12}, v_{3}, v_{41}>+<v_{2},-v_{12},-v_{4}, v_{41}>\text {. }
$$

The maps $\psi^{(3)}$ sends it to the boundary of Voronoi's 5-simplex $\varphi\left(v_{1}, v_{2}, v_{3}\right.$, $\left.v_{4}, v_{12}, v_{23}\right)$ :

$$
\begin{array}{r}
\varphi\left(v_{1}, v_{2}, v_{3}, v_{4}, v_{12}\right)-\varphi\left(v_{1}, v_{2}, v_{3}, v_{4}, v_{23}\right)+\varphi\left(v_{1}, v_{12}, v_{3}, v_{23}, v_{2}\right)- \\
-\varphi\left(v_{1}, v_{12}, v_{3}, v_{23}, v_{4}\right)+\varphi\left(v_{2}, v_{12}, v_{4}, v_{23}, v_{1}\right)-\varphi\left(v_{2}, v_{12}, v_{4}, v_{23}, v_{3}\right)= \\
d \varphi\left(v_{1}, v_{2}, v_{3}, v_{4}, v_{12}, v_{23}\right) .
\end{array}
$$


The other components of the map $\psi^{(3)}$ are obviously group homomorphisms. The map $\psi^{(3)}$ respects the differentials, and it is clearly injective. It is an isomorphism in all the degrees except 3 and 4 . One has

$$
\operatorname{Coker}\left(\psi^{(3)}\right)=\frac{V_{4}^{(3)}}{\psi^{(3)}\left(M_{4}^{(3)}\right)} \stackrel{\partial}{\longrightarrow} \frac{V_{3}^{(3)}}{\psi^{(3)}\left(M_{3}^{(3)}\right)} .
$$

Denote by $\left\{v_{1}, v_{2}, v_{3}, v_{4}\right\}$ the set of all unordered 4-tuple of vectors $\left(v_{1}, v_{2}, v_{3}, v_{4}\right)$ in $L_{3}$ such that $v_{1}+v_{2}+v_{3}+v_{4}=0$ and $\left(v_{1}, v_{2}, v_{3}\right)$ is a basis of $L_{3}$.

Proposition 6.3. One has canonical isomorphisms

$$
\frac{V_{4}^{(3)}}{\psi^{(3)}\left(M_{4}^{(3)}\right)}=\mathbb{Z}\left[\left[\left\{v_{1}, v_{2}, v_{3}, v_{4}\right\}\right]\right]=\frac{V_{3}^{(3)}}{\psi^{(3)}\left(M_{3}^{(3)}\right)} .
$$

It transforms the differential in (31) to the identity map on $\mathbb{Z}\left[\left[\left\{v_{1}, v_{2}, v_{3}, v_{4}\right\}\right]\right]$. Therefore the complex Coker $\left(\psi^{(3)}\right)$ is acyclic.

Proof. The following observations about the Voronoi cell decomposition are easy to see from figure 4 .

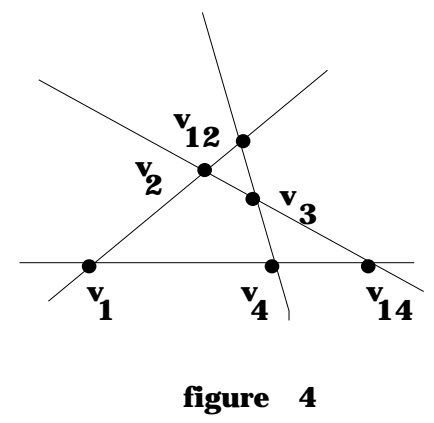

1) A 3-dimensional cell of Voronoi's decomposition is $G L_{3}(\mathbb{Z})$-equivalent to one of the following two: a 3-cell $\varphi\left(v_{1}, v_{2},-v_{12}, v_{3}\right)$, called special 3-cell, or a 3 -cell $\varphi\left(v_{1}, v_{2}, v_{3}, v_{4}\right)$, called generic 3 -cell. So generic 3 -cells are parametrized by the set $\left\{v_{1}, v_{2}, v_{3}, v_{4}\right\}$.

2) A 5 -simplex containing generic 3 -cell $\varphi\left(v_{1}, v_{2}, v_{3}, v_{4}\right)$ is determined by the dihedral order of the vectors $v_{1}, v_{2}, v_{3}, v_{4}$. So a given generic 3 -cell is contained in three 5 -simplices. For the 3 -cell $\varphi\left(v_{1}, v_{2}, v_{3}, v_{4}\right)$ these are

$$
\varphi\left(v_{1}, v_{2}, v_{3}, v_{4}, v_{12}, v_{23}\right), \quad \varphi\left(v_{2}, v_{1}, v_{3}, v_{4}, v_{21}, v_{13}\right), \quad \varphi\left(v_{2}, v_{3}, v_{1}, v_{4}, v_{23}, v_{31}\right) .
$$

3) The elements (30) are in bijective correspondence with the pairs \{generic 3-cell, a 5-cell containing it\}. The right hand side of (30) is the sum of the 4-cells containing a given generic 3-cell and contained in a given 5-cell.

The first shuffle relation means that the sum of the elements of type (30) corresponding to 5-cells containing a given generic 3-cell is zero. 
4) A given 5-simplex has only three generic 3-cells. For the 5-simplex $\varphi\left(v_{1}, v_{2}, v_{3}, v_{4}, v_{12}, v_{23}\right)$ they are

$$
\varphi\left(v_{1}, v_{2}, v_{3}, v_{4}\right), \quad \varphi\left(v_{12}, v_{4}, v_{23},-v_{2}\right), \quad \varphi\left(v_{12}, v_{3},-v_{23},-v_{1}\right) .
$$

The sum of the elements (30) corresponding to generic 3-cells of a given 5-simplex is the second shuffle relation. It is the boundary of that 5 -simplex.

The observation 1) implies the second isomorphism in (32). The observations 2 ) and 3) lead to the first isomorphism in (32). It is easy to check that the differential is the identity map. The proposition is proved.

\section{Applications to multiple $\zeta$-values}

1. Regularization and the map $\mathcal{D}_{w, m}(N) \longrightarrow \bar{Z}_{w, m}(N)$. The iterated integral $I_{n_{1}, \ldots, n_{m}}\left(a_{1}: \ldots: a_{m}: 1\right)$ is divergent if and only if $n_{m}=1, a_{m}=1$. The power series $L i_{n_{1}, \ldots, n_{m}}\left(x_{1}, \ldots, x_{m}\right)$ are divergent if and only if $n_{m}=1, x_{m}=1$.

Theorem 7.1. a) Let $\left|x_{i}\right| \leq 1$. Then both the power series

$$
L i_{n_{1}, \ldots, n_{l}, 1, \ldots, 1}\left(x_{1}, \ldots, x_{l}, 1-\varepsilon, \ldots, 1-\varepsilon\right)
$$

and the power series $L i_{n_{1}, \ldots, n_{l}, 1, \ldots, 1}\left(x_{1}, \ldots, x_{l}, 1, \ldots, 1,1-\varepsilon\right)$ corresponding to

$$
I_{n_{1}, \ldots, n_{l}, 1, \ldots, 1}\left(a_{1}: \ldots: a_{l}: 1: \ldots: 1: 1-\varepsilon\right)
$$

admit asymptotic expansions which are polynonials in $\log \varepsilon$. Their coefficients are explicitely computable sums of multiple polylogarithms.

b) The constant terms of these expansions differ by lower depth multiple polylogarithms, and the other terms are of lower depth. In particular if $x_{i}^{N}=1$ they define the same element $\operatorname{Reg} L i_{n_{1}, \ldots, n_{m}}\left(x_{1}, \ldots, x_{m}\right) \in \bar{Z}_{\bullet \bullet \bullet}(N)$.

c) The map $\left\{x_{1}, \ldots, x_{m}\right\}_{n_{1}, \ldots, n_{m}} \longmapsto \operatorname{Reg} L i_{n_{1}, \ldots, n_{m}}\left(x_{1}, \ldots, x_{m}\right)$ provides $a$ surjective linear map $\mathcal{D}_{w, m}(N) \stackrel{\longrightarrow}{\longrightarrow} \bar{Z}_{w, m}(N)$.

Proof. Direct integration gives us

$$
I_{1, \ldots, 1}(1: \ldots: 1: 1-\varepsilon)=\int_{0<t_{1}<\ldots<t_{m}<1-\varepsilon} \frac{d t_{1}}{1-t_{1}} \wedge \ldots \wedge \frac{d t_{m}}{1-t_{m}}=\frac{(-\log \varepsilon)^{m}}{m !} .
$$

Computing $\left(\sum_{k>0} \frac{(1-\varepsilon)^{k}}{k}\right)^{m}$ and using then induction in $m-l$ we get

$$
\begin{aligned}
\frac{(-\log \varepsilon)^{m}}{m !}=\quad L i_{1, \ldots, 1}(1-\varepsilon, & \ldots, 1-\varepsilon) \\
& \left.+\sum_{0<i<m} \text { (lower depth multiple } \zeta^{\prime} \text { 's }\right)(\log \varepsilon)^{i} .
\end{aligned}
$$

Assume $n_{l} \neq 1$ or $x_{l} \neq 1$. Applying the power series product formula to

$$
L i_{n_{1}, \ldots, n_{l}}\left(x_{1}, \ldots, x_{l}\right) \cdot L i_{1, \ldots, 1}(1-\varepsilon, \ldots, 1-\varepsilon)
$$

and then to

$$
L i_{n_{1}, \ldots, n_{l}}\left(x_{1}, \ldots, x_{l}\right) \cdot L i_{1, \ldots, 1}(1, \ldots, 1,1-\varepsilon)
$$


and using the induction on $m-l$, we get parts a) and b) of the theorem.

c) The power series (resp. the iterated integral) product formulas clearly hold for the asymptotic expansions (33) (resp. (34)). So the shuffle relations are valid. The distribution relations for $l>0$ hold for (33). The distribution relations for $l=-1$ follows from the shuffle relations and theorem 4.1 .

Corollary 7.2. $\operatorname{dim} \bar{Z}(1)_{w, m}=0$ if $w+m$ is odd.

Proof. Indeed, (21) implies that $\operatorname{dim} \mathcal{D}(1)_{w, m}=0$ if $w+m$ is odd.

2. Proof of theorem 1.3. It follows from theorems 1.2, 6.1 and 7.1.

3. Proof of theorem 1.4. If $w>2$ then $H^{0}\left(G L_{2}(\mathbb{Z}), S^{w-2} V_{2}\right)=0$, and

$$
\sum_{w} \operatorname{dim} H^{1}\left(G L_{2}(\mathbb{Z}), S^{w-2} V_{2}\right) \cdot t^{w}=\frac{1}{\left(1-t^{4}\right)\left(1-t^{6}\right)}-1
$$

So by (10) the generating function for the Euler characteristic of the complex $\mathcal{D}_{\bullet, 2} \longrightarrow \Lambda^{2} \mathcal{D}_{\bullet, 1}$ is given by (35). Using formula (12) we get the result.

Proof of theorem 1.5. Since $S^{w-3} V_{3}$ for $w>3$ is not a self dual $G L_{3}$-module, the kernel of the restriction of $H^{i}\left(G L_{3}(\mathbb{Z}), S^{w-3} V_{3}\right)$ to the boundary of the Borel-Serre bordification vanishes by a theorem of Borel [BW]. Computing the boundary contribution to the cohomology we get

$$
H^{i}\left(G L_{3}(\mathbb{Z}), S^{w-3} V_{3}\right)= \begin{cases}0 & i=1,2 \\ H_{\mathrm{usp}}^{1}\left(G L_{2}(\mathbb{Z}), S^{w-2} V_{3}\right) & i=3\end{cases}
$$

Combining formulas (11) and (36) we get the cohomology of the complex $\mathcal{D}_{\bullet, 3} \longrightarrow \mathcal{D}_{\bullet, 2} \otimes \mathcal{D}_{\bullet, 1} \longrightarrow \Lambda^{3} \mathcal{D}_{\bullet, 1}$. Using theorems 1.4 and 7.1 , formula (12), and the Euler characteristic argument, we obtain the theorem.

\section{Acknowledgments}

I would like to thank the Max-Planck-Institute (Bonn) and University ParisXI (Orsay) for hospitality and support.

I am grateful to J. Bernstein, G. Frey, G. Harder, M. Kontsevich, B. B. Venkov, V. Voevodsky, D. Zagier for useful and stimulating discussions.

\section{References}

[A] A. Ash, Cohomolgy of congruence subgroups of $S L(3, \mathbb{Z})$, Math. Ann. 249 (1980), 55-73.

[BGSV] A. A. Beilinson, A. B. Goncharov, V. V. Schechtman, and A. N. Varchenko, Aomoto dilogarithms, mixed Hodge structures and motivic cohomolgy, Grothendieck Feschtrift, Birkhouser, vol. 86, 1990, pp. 135-171.

[B] A. Borel and N. Wallach, Continuous cohomology, discrete subgroups and representations of reductive groups, Ann. of Math. Studies, 94, 1980.

[B] D. J. Broadhurst, On the enumeration of irreducible $k$-fold sums and their role in knot theory and field theory, Preprint hep-th/9604128.

[E] L. Euler, "Opera Omnia”, Ser. 1, Vol XV, Teubner, Berlin 1917, 217-267. 
[G1] A. B. Goncharov, Multiple $\zeta$-numbers, hyperlogarithms and mixed Tate motives, Preprint MSRI 058-93, June 1993.

[G2] _ Polylogarithms in arithmetic and geometry, Proc. ICM-94, Zurich, 1995, pp. 374-387.

[G3] , The double logarithm and Manin's complex for modular curves, Math. Res. Lett., 4 (1997), 617-636.

[G4] _ Multiple polylogarithms at roots of unity and motivic Lie algebras, in Preprint MPI-62/97, Proc. of Arbeitstagung, Bonn, June 1997.

[G4] Mixed elliptic motives, Proc. of 60-th Durham symposium "Galois groups in arithmetic algebraic geometry", 1998.

[D] P. Deligne, Le group fondamental de la droite projective moine trois points, In: Galois groups over $\mathbb{Q}$. Publ. MSRI, no. 16, (1989), 79-298.

[Dr] V. G. Drinfeld, On quasi-triangular quasi-Hopf algebras and some group related to $\operatorname{Gal}(\overline{\mathbb{Q}} / \mathbb{Q})$, Leningrad Math. J. 2 (1991), 829-860.

[Ih] Y. Ihara, Braids, Galois groups, and some arithmetic functions, Proc. Int. Congress of Mathematicians, Vol. I, II (Kyoto, 1990), 99-120, Math. Soc. Japan, Tokyo, 1991.

[Ih1] The Galois representation arising from $\mathbb{P}^{1} \backslash\{0,1, \infty\}$ and Tate twists of even degree, Galois groups over $Q$ (Berkeley, CA, 1987), 299-313, Math. Sci. Res. Inst. Publ., 16, Springer, New York-Berlin, 1989.

[Kr] D. Kreimer, Renormalisation and knot theory, J. Knot Theory Ramifications 6 (1997), 479-581.

[M] J. Martinet, Les reseaux parfaits des espaces euclidiens, Mathématiques, Masson, Paris, 1996.

[V] G. Voronoi, Nouvelles applications des paramétres continus á la théorie des formes quadratiques, I, J. Reine Angew. Math. 133 (1908), 97-178.

[Z1] D. Zagier, Values of zeta functions and their applications, Proceedings of the First Europian Congress of Mathematicians, Paris. 1994, vol 1.

[Z2] _ Periods of modular forms, traces of Hecke operators, and multiple zeta values, RIMS Kokyuroku 843 (1993), 162-170.

Department of Mathematics, Brown University, Providence, Ri 02912

E-mail address: sasha@math.brown.edu 